\title{
Efficacy of Topical Compound Danxiong Granules for Treatment of Dermatologic Toxicities Induced by Targeted Anticancer Therapy: A Randomized, Double-Blind, Placebo-Controlled Trial
}

\author{
Aiping Tian, ${ }^{1}$ Aiping Zhou, ${ }^{2}$ Xinyu Bi, ${ }^{3}$ Shangying Hu, ${ }^{4}$ Zhichao Jiang, ${ }^{2}$ Wen Zhang, \\ Zhen Huang, ${ }^{3}$ Hongzhe Shi, ${ }^{5}$ Boyan Yang, ${ }^{6}$ and Wei Chen ${ }^{7}$ \\ ${ }^{1}$ Department of Traditional Chinese Medicine, National Cancer Center, Cancer Hospital, Chinese Academy of Medical Sciences \\ and Peking Union Medical College, Beijing 100021, China \\ ${ }^{2}$ Department of Medical Oncology, National Cancer Center, Cancer Hospital, Chinese Academy of Medical Sciences \\ and Peking Union Medical College, Beijing 100021, China \\ ${ }^{3}$ Department of Hepatobiliary Surgery, National Cancer Center, Cancer Hospital, Chinese Academy of Medical Sciences \\ and Peking Union Medical College, Beijing 100021, China \\ ${ }^{4}$ Department of Cancer Epidemiology, National Cancer Center, Cancer Hospital, Chinese Academy of Medical Sciences \\ and Peking Union Medical College, Beijing 100021, China \\ ${ }^{5}$ Department of Urology, National Cancer Center, Cancer Hospital, Chinese Academy of Medical Sciences and Peking Union \\ Medical College, Beijing 100021, China \\ ${ }^{6}$ Emergency Department, National Cancer Center, Cancer Hospital, Chinese Academy of Medical Sciences and Peking Union \\ Medical College, Beijing 100021, China \\ ${ }^{7}$ Department of Pharmacy, National Cancer Center, Cancer Hospital, Chinese Academy of Medical Sciences and Peking Union \\ Medical College, Beijing 100021, China \\ Correspondence should be addressed to Aiping Tian; aipingtian@126.com
}

Received 15 March 2017; Revised 23 June 2017; Accepted 2 July 2017; Published 6 August 2017

Academic Editor: Jenny M. Wilkinson

Copyright (C) 2017 Aiping Tian et al. This is an open access article distributed under the Creative Commons Attribution License, which permits unrestricted use, distribution, and reproduction in any medium, provided the original work is properly cited.

Dermatologic toxicities resulting in dose reduction or discontinuation of treatment pose challenges for targeted anticancer therapies. We conducted this randomized, double-blind, placebo-controlled trial to investigate the efficacy of topical application of Compound Danxiong Granules (CDG) for treatment of dermatologic toxicities associated with targeted anticancer therapies. One hundred and ten patients with dermatologic toxicities induced by targeted anticancer therapies were randomly assigned to CDG or placebo group. Each crude herb (Rhizoma Chuanxiong, Paeonia suffruticosa Andr., Cortex Phellodendri, Geranium sibiricum L., and Flos Carthami) was prepared as an instant herbal powder. Application of the CDG via topical washes lasted 20 minutes, twice daily, for 10 days. The primary outcome was the total effective rate, defined as reduction in at least one grade of skin toxicity. The total effective rate was $77.61 \%(52 / 67)$ in the CDG group and $27.27 \%(9 / 33)$ in the placebo group $(P<0.0001)$. Compared to the placebo treatment, CDG treatment achieved a higher total effective rate for hand-foot skin reaction (95.45\% versus $27.27 \%)$, acneiform eruption (69.23\% versus $30.78 \%$ ), and paronychia (68.42\% versus $22.22 \%)$. Topical application of CDG can effectively attenuate dermatologic toxicities induced by targeted anticancer therapies. The effect of CDG was more pronounced in hand-foot skin reaction.

\section{Introduction}

Targeted anticancer therapies have improved survival outcomes in various cancer patients [1]. Anticancer agents target signal transduction, tumor angiogenesis, or tumor microenvironment pathways [2]. Introduction of these agents has been associated with a wide spectrum of dermatologic toxicities [3]. Cutaneous adverse reactions associated with targeted anticancer therapies commonly manifest as acneiform eruption, hand-foot skin reaction, paronychia, 
skin fissures, and dermal hypersensitivity reaction [4]. Despite the fact that these dermatologic toxicities are not life threatening, dose reduction or discontinuation of cancer treatment and impairments in patient quality of life pose a great challenge for targeted cancer therapies [5]. Consequently, management of these dermatologic toxicities further increases the economic burden of cancer care [6]. However, mechanisms underlying the targeted anticancer therapiesassociated cutaneous toxicities remain incompletely characterized. Interference with the follicular and interfollicular epidermal growth signaling pathway is thought to be critical [7]. Therefore, unified standardized therapy for management of the targeted anticancer therapies associated with dermatologic toxicities is lacking [8]. Currently, the primary therapies to treat dermatologic symptoms are antibody, antiallergic, and local supportive treatments [9], but these agents are not highly effective. Therefore, there is an urgent need to find an alternative therapeutic agent to prevent and manage dermatologic toxicities induced by targeted anticancer therapies.

Traditional Chinese Medicine (TCM) has long been used for the treatment of dermatologic disorders $[10,11]$ and cancer $[12,13]$. Thus, consideration should be given to the beneficial effects of Chinese herbal prescription for management of dermatologic toxicities induced by targeted anticancer therapies. Many TCM practitioners have attempted to use Chinese herbs for management of targeted agent-related dermatologic toxicities [14]. Topical applications are the most accessible type of herbal treatment. However, evidence of topical use of Chinese herbal prescription in management of dermatologic toxicities induced by anticancer therapies was mainly based on case series, physician experiences, or self-controlled studies. Thus, there is a lack of well-designed double-blind, placebo-controlled trials to support the evidence.

The objective of this trial was to investigate the efficiency of Compound Danxiong Granules (CDG) in the treatment of dermatologic toxicities induced by targeted anticancer therapies.

\section{Methods}

2.1. Study Design and Patients. This randomized, doubleblind, placebo-controlled trial was conducted in the Department of Traditional Chinese Medicine at the National Cancer Center/Cancer Hospital, Chinese Academy of Medical Sciences and Peking Union Medical College, from September 2010 to July 2014. The Ethics Committee of Cancer Hospital, Chinese Academy of Medical Sciences, approved the study protocol. Written informed consent was obtained from all patients before enrollment. Patient eligibility was based on the following inclusion criteria: (1) aged 18 years or over; (2) an Eastern Cooperative Oncology Group performance status $\leq 3$; (3) malignant tumor diagnosed by evidence of pathological or cytological findings; (4) treatment with targeted anticancer agents and occurrence of dermatologic toxicities including acneiform eruption, paronychia, and hand-foot skin reaction; and (5) not receiving other preparations of traditional herbal medicines during the 4 weeks before enrollment. The exclusion criteria were (1) dermatologic toxicities not induced by targeted anticancer agents; (2) concurrent acne vulgaris, eczema, psoriasis, and other skin diseases; (3) skin hypersensitiveness; and (4) intellectual and mental disorders, abnormal language expression ability, and an inability to judge or express their own symptoms.

2.2. Preparation of $C D G$ and Placebo. This study utilized five Chinese herbs: Paeonia suffruticosa Andr. (20 g), Rhizoma Chuanxiong (18g), Flos Carthami (12 g), Cortex Phellodendri $(18 \mathrm{~g})$, and Geranium sibiricum L. $(20 \mathrm{~g})$. The detailed characteristics of the constituents in CDG are summarized in Table 1. All crude herbs were purchased from China Resources Sanjiu Medical \& Pharmaceutical Co., Ltd., and were prepared as an instant herbal powder. The cotton bag containing the above instant herbal powder was immersed in $60-70^{\circ} \mathrm{C}$ water and then diluted in $1,000 \mathrm{ml}$ water. The bag was stirred to accelerate release of the effective ingredients until the temperature dropped to $25-30^{\circ} \mathrm{C}$. Placebo powder was made from dextrin and edible pigment and had the same color and appearance as CDG.

2.3. Intervention. Eligible patients were randomly allocated $2: 1$ to either a CDG $(n=73)$ or placebo $(n=37)$ group using a computer-generated randomization code. The investigators were blinded to the sequence code until the trial was completed. The tested agents were supplied in labeled packaged containers and provided to the patients and the investigators in a double-blind manner. Patients were instructed to use their prescribed preparation before treatment. Patients were asked to start topical application of their tested drugs after random grouping. For hand-foot skin reaction and paronychia patients, the skin lesions were soaked in the herbal liquid. For acneiform eruption in the facial and trunk cadre patients, a gauze patch with herbal liquid was repeatedly applied to the skin lesions. A dosage of CDG and placebo was used as a topical wash for 20 minutes, twice daily, until the water temperature dropped to below $25^{\circ} \mathrm{C}$. Treatment duration lasted for 10 consecutive days.

2.4. Assessment of Dermatological Toxicities. Rash severity was graded according to the US National Cancer Institute Common Toxicity Criteria for Adverse Events version 3.0 (NCI CTCAE v3.0) [15] and by consensus among Chinese oncologist experts: Grade I: localized rash (mainly face and upper trunk) without associated symptoms, without interfering with daily activities, and with no signs of secondary infection; Grade II: scattered, but not generalized eruption, slightly interfering with daily activities, and without secondary infection signs; and Grade III: widespread rash with severe objective symptoms, interfering with daily activities, and associated with local secondary infection. The severity of nail changes was graded according to the NCI CTCAE v3.0: Grade I: nail decolorization and wrinkles, without disruption of daily activities; Grade II: nail partially or completely detached and painful nail plate; and Grade III: severe nail plate lesion (paronychia) interfering with daily activities and/or secondary infection. Hand-foot skin reaction was also graded in accordance with the NCI CTCAE v3.0: Grade I: minimal skin changes or dermatitis (e.g., erythema and 
TABLE 1: Constituents in the Compound Danxiong Granules.

\begin{tabular}{|c|c|c|c|c|c|c|}
\hline Chinese name & English herb name & Latin herb name & Family & Species & TCM action & Dose used \\
\hline Chuān xiōng & $\begin{array}{l}\text { Szechuan lovage } \\
\text { Rhizome }\end{array}$ & Rhizoma Chuanxiong & Apiaceae & $\begin{array}{l}\text { Ligusticum chinense } \\
\text { (L.) Crantz }\end{array}$ & Blood-activating & $18 \mathrm{~g}$ \\
\hline Mǔ dān pí & Tree Peony Bark & $\begin{array}{c}\text { Paeonia suffruticosa } \\
\text { Andr. }\end{array}$ & Paeoniaceae & P. suffruticosa & $\begin{array}{l}\text { Cooling the blood } \\
\text { and detoxicating }\end{array}$ & $20 \mathrm{~g}$ \\
\hline Huáng bò & $\begin{array}{c}\text { Amur Corktree } \\
\text { Bark }\end{array}$ & Cortex Phellodendri & Rutaceae & $\begin{array}{l}\text { Phellodendron } \\
\text { chinense Schneid. }\end{array}$ & $\begin{array}{l}\text { Clearing heat and } \\
\text { dampness }\end{array}$ & $18 \mathrm{~g}$ \\
\hline Lǎo Guàn Cǎo & Cranesbill Herb & Geranium sibiricum L. & Geraniaceae & Geranium & $\begin{array}{l}\text { Clearing dampness } \\
\text { and blood-activating }\end{array}$ & $20 \mathrm{~g}$ \\
\hline Hóng huā & Safflower & Flos Carthami & Compositae & $\begin{array}{c}\text { Carthamus tinctorius } \\
\text { L. }\end{array}$ & Blood-activating & $12 \mathrm{~g}$ \\
\hline
\end{tabular}

scaling) without pain; Grade II: skin changes (e.g., peeling, blisters, bleeding, and edema) or pain, not interfering with daily activities; and Grade III: ulcerative dermatitis or skin changes with pain interfering with daily activities.

2.5. Outcome Measures. The primary end point was the total effective rate. "Effective" indicated a reduction in at least one grade in dermatological toxicity. "Cure" was defined as the absence of skin lesions. "Treatment failure" indicated no improvement in grade of dermatological toxicity. The total effective rate was defined as the number of patients who achieved "Effective" (reduction in at least one grade of dermatological toxicity) divided by the total number of patients. The secondary end point was safety determined by routine blood tests and measurements of liver and renal function. Physicians were trained to grade dermatitis before the beginning of this trial. Dermatological toxicities were evaluated at baseline and at the end of treatment by experienced oncologists and dermatologists. All investigators took photographs using a CANON Digital IXUS 500 at enrollment and after 10 days of treatment. Photographs were used with patient authorization. Routine blood tests and liver and renal function were determined before and after 10 days of treatment. The following data were also collected for each patient: type of tumor, targeted anticancer agents, skin lesion type, severity of skin lesions, and ongoing, targeted anticancer therapy.

2.6. Statistical Analysis. The sample size was calculated to ensure specific significance, during which statistical power was taken as $90 \%$ at the $5 \%$ significance level. Statistically significant increases in the total effective rate at $25 \%$ were used for this purpose based on our pilot study comparison of the total effective rate of the CDG group (60\%) and the control group (35\%). Finally, considering a 10\% dropout rate, our study required a total of 111 patients. All statistical analyses were carried out using SPSS 19.0 software (SPSS Inc., Chicago, IL, USA). Categorical data are expressed as frequencies and percentages. Comparisons of categorical variables between the CDG and placebo groups were analyzed using the Chisquare test. The Fisher exact test was utilized. A $P$ value < 0.05 was considered statistically significant.

\section{Results}

3.1. Baseline Characteristics. Figure 1 shows the flow diagram of patient enrollment and randomization through the study. A total of 110 eligible patients were randomized and 100 patients completed planned topical wash therapy and assessment. Ten patients discontinued topical washing treatment. The reasons for discontinuation of topical washing treatment were spontaneous pain relief $(n=2)$ and being lost to followup $(n=3)$. Discontinuation of anticancer treatment resulted from severe hypertension, liver injury, or disease progression $(n=5)$. Table 2 shows the baseline characteristics of the two treatment groups. The most frequently reported skin toxicities among the included patients were acneiform eruption (39.0\%), hand-foot skin reaction $(33.0 \%)$, and paronychia (28.0\%). There was no significant difference between the two groups with regard to age or gender distribution, tumor type, type of skin lesions, targeted anticancer agents, and severity of skin lesions (all $P>0.05$ ).

3.2. Comparison of Total Clinical Effect. As shown in Table 3, treatment was effective in $52(77.61 \%)$ cases in the CDG group and $9(27.27 \%)$ cases in the placebo group, with a statistically significant difference between the two groups (Chi-square = $23.55, P<0.001)$. The clinical cure rate was $12 / 67$ patients $(17.91 \%)$ in the CDG group and no patients achieved a clinical cure in the placebo group.

3.3. Comparison of the Clinical Effect on Different Types of Skin Lesions. As shown in Table 4, the treatment for handfoot skin reaction, acneiform eruption, and paronychia was effective in 21 (95.45\%), 18 (69.23\%), and $13(68.42 \%)$ cases in the CDG group, respectively. In contrast, the treatment for hand-foot skin reaction, acneiform eruption, and paronychia was effective in $3(27.27 \%), 4$ (30.78\%), and $2(22.22 \%)$ cases in the placebo group, respectively. There were statistically significant differences regarding hand-foot skin reaction, acneiform eruption, and paronychia between the two groups $(P<0.05)$. The clinical cure rate in CDG treated patients was $31.82 \%$ for hand-foot skin reaction, $11.54 \%$ for acneiform eruption, and $10.53 \%$ for paronychia. Before and after CDG treatment, images are presented in Figure 2. 
TABLE 2: Baseline patient characteristics.

\begin{tabular}{|c|c|c|c|}
\hline Characteristic & $\begin{array}{l}\text { Compound } \\
\text { Danxiong } \\
\text { Granules } \\
(n=67)\end{array}$ & $\begin{array}{l}\text { Placebo } \\
(n=33)\end{array}$ & $P$ value \\
\hline Mean age/range (years) & $55(31-72)$ & $53(33-70)$ & 0.478 \\
\hline Sex & & & 0.841 \\
\hline Male & $44(65.7 \%)$ & $21(63.6 \%)$ & \\
\hline Female & $23(24.3 \%)$ & $12(26.4 \%)$ & \\
\hline Type of tumor & & & 0.992 \\
\hline Hepatocarcinoma & $12(17.9 \%)$ & $5(15.2 \%)$ & \\
\hline Renal carcinoma & $10(14.9 \%)$ & $6(18.2 \%)$ & \\
\hline Lung cancer & $22(32.8 \%)$ & $11(33.3 \%)$ & \\
\hline Intestinal cancer & $21(31.3 \%)$ & $10(30.3 \%)$ & \\
\hline Breast cancer & $1(1.5 \%)$ & $1(3.0 \%)$ & \\
\hline Soft tissue sarcoma & $1(1.5 \%)$ & & \\
\hline $\begin{array}{l}\text { Targeted anticancer } \\
\text { agents }\end{array}$ & & & 0.997 \\
\hline Sorafenib & $14(20.9 \%)$ & $6(18.2 \%)$ & \\
\hline Sunitinib & $8(11.9 \%)$ & $5(15.2 \%)$ & \\
\hline Erlotinib & $12(17.9 \%)$ & $6(18.2 \%)$ & \\
\hline Gefitinib & $10(14.9 \%)$ & $5(15.2 \%)$ & \\
\hline Cetuximab & $21(31.3 \%)$ & $10(30.3 \%)$ & \\
\hline Famitinib & $2(3.0 \%)$ & $1(3.0 \%)$ & \\
\hline Type of skin lesions & & & 0.994 \\
\hline $\begin{array}{l}\text { Hand-foot skin } \\
\text { reaction }\end{array}$ & $22(32.8 \%)$ & $11(33.3 \%)$ & \\
\hline Acneiform eruption & $26(38.8 \%)$ & $13(39.4 \%)$ & \\
\hline Paronychia & $19(28.9 \%)$ & $9(27.3 \%)$ & \\
\hline Severity of skin lesions & & & 0.134 \\
\hline Grade I & $5(7.5 \%)$ & $6(18.2 \%)$ & \\
\hline Grade II & $28(41.8 \%)$ & $16(48.5 \%)$ & \\
\hline Grade III & $34(50.7 \%)$ & $11(33.3 \%)$ & \\
\hline $\begin{array}{l}\text { Time after the initiation } \\
\text { of targeted anticancer } \\
\text { therapies }\end{array}$ & $23 \pm 10$ (days) & $24 \pm 14$ (days) & 0.700 \\
\hline
\end{tabular}

3.4. Incidence of Discontinuation of Cancer Treatment. Discontinuation of cancer treatment was reported in $6(9.96 \%)$ patients in the CDG group and $4(12.1 \%)$ patients in the placebo group. There was no significant difference with regard to the incidence of discontinuation of cancer treatment between the two treatment groups $(P=0.887)$. Discontinuation of targeted anticancer therapy was not correlated with skin toxicity. Adjustment of targeted anticancer agents and/or liver or cardiovascular toxicities caused by the targeted therapies were reasons for discontinuation of cancer treatment.

3.5. Adverse Effects. The skin treatment regimen was well tolerated and did not cause any adverse effects. The routine blood tests and biochemical analyses did not differ when comparing before and after herbal treatment.

\section{Discussion}

This randomized, double-blind, placebo-controlled trial demonstrated that topical use of CDG, as an instant herbal powder, was significantly effective in minimizing dermatologic toxicities induced by targeted anticancer therapies. More importantly, the cure rate $(31.82 \%)$ and total effective rate $(95.45 \%)$ of hand-foot skin reaction were more pronounced in CDG treated patients compared to patients in the placebo group.

In this study, the incidence of dermatologic toxicities was $31 \%$ for Cetuximab, $20 \%$ for Sorafenib, and $18 \%$ for Erlotinib. The most frequently encountered dermatologic toxicities were acneiform eruption (39\%), hand-foot skin reaction $(33 \%)$, and paronychia $(28 \%)$. The total effective rate in the CDG group was significantly higher than that of the placebo group (77.61\% versus $27.27 \%$, resp.). The effect of CDG on hand-foot skin reaction was particularly more pronounced, and nearly one-third of these patients with Grade II or higher skin lesions achieved clinical cure. The painful symptoms and skin lesions were attenuated during days 3-10 following treatment.

There are many approaches for the management of dermatologic toxicities induced by targeted anticancer therapies [9]. Early intervention is critical in treating dermatologic toxicities. Appropriate and timely management of dermatologic toxicities may prevent dose alteration or discontinuation of cancer treatment. A well-designed systematic review indicated that antibiotics were the most common treatment option and had the potential to reduce the severity of epidermal growth factor receptor inhibitor-induced skin toxicities [16]. Unfortunately, a unified standard therapy based on these studies is still lacking.

Traditional Chinese herbal therapies have demonstrated benefits for management of dermatologic toxicities induced by targeted anticancer agents [17]. An early randomized controlled trial (RCT) [18] showed that antipruritic prescription (Radix Stemonae, Xanthium sibiricum, clove, lotus leaf, honeysuckle, Sophora flavescens Ait., Portulaca oleracea, and dwarf lilyturf) was superior to calamine lotion in attenuating acneiform eruption caused by targeted anticancer agents. Another RCT [19] demonstrated that external application of Zhiyang Pingfu Liquid significantly attenuated itching, rash, and acneiform eruptions induced by epidermal growth factor receptor inhibitors compared with erythromycin ointment. Zhiyang Pingfu Liquid formula is composed of Scutellaria baicalensis, Sophora flavescens, Portulaca oleracea, and bark of Dictamnus dasycarpus. Zhang et al. [20] investigated the clinical efficacy of the Zhiyang Pingfu Liquid formula as an oral treatment combined with topical administration of Yangfei Xiaozhen Formula (coastal glehnia root, Ophiopogon japonicus, Schisandra chinensis, honeysuckle, Sophora flavescens, bark of Dictamnus dasycarpus, cortex moutan, etc.) for treating rash induced by lung cancer targeted agents in 80 patients. They found that the total curative effect of rash grade was 75\% in the patients receiving Yangfei Xiaozhen Formula combined with external therapy and 55\% in the patients receiving external therapy alone $(P<0.05)$. Moreover, oral 


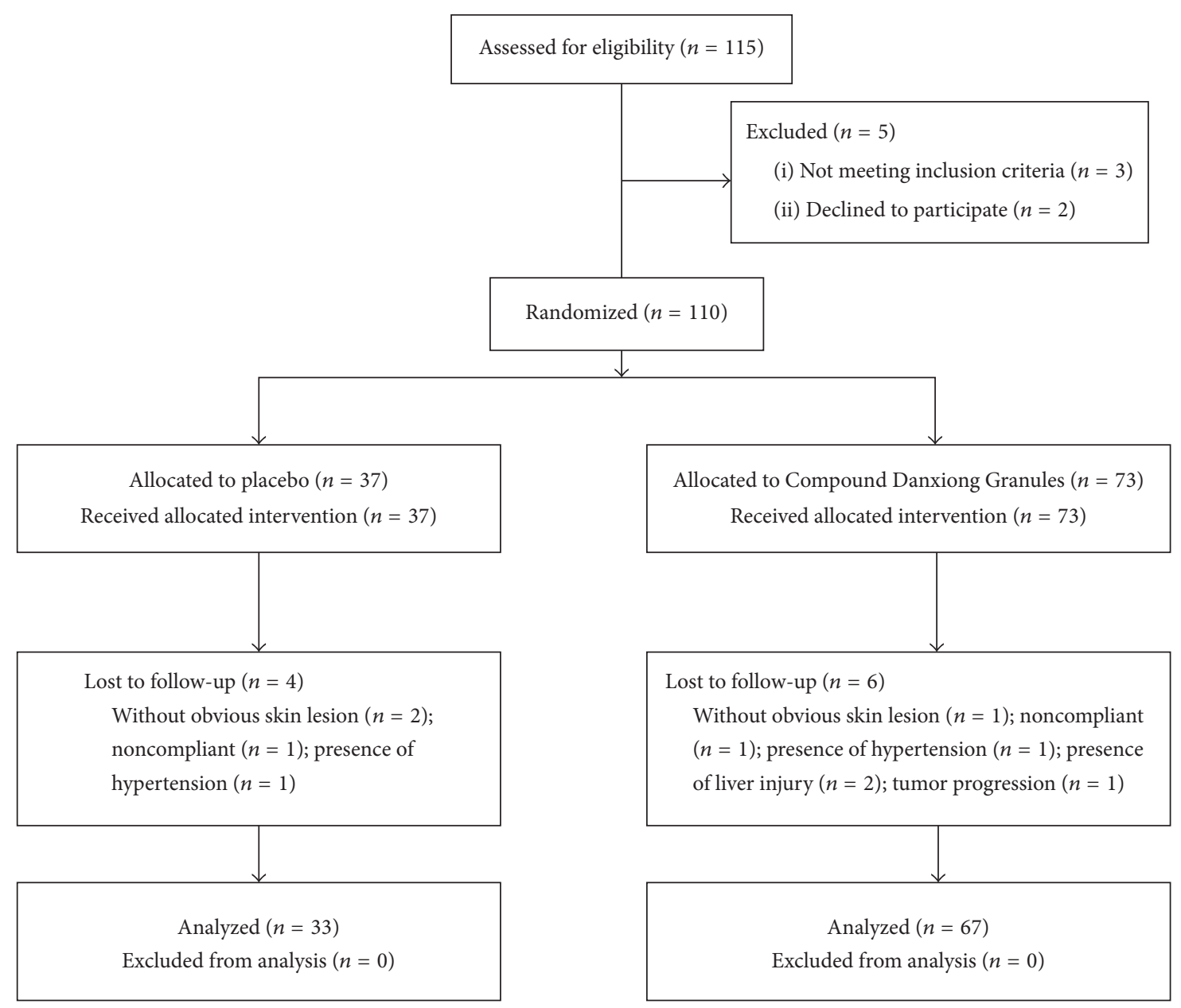

FIGURE 1: Flow diagram of patient enrollment and randomization.

Yangfei Xiaozhen Formula significantly improved the quality of life compared to the control group.

Chinese herbal therapies for dermatologic toxicities can be divided into two types of oral and topical medicines. Topical use is the most commonly prescribed intervention for dermatologic patients. Topically applied herbal lotion lowers the first pass effect through the liver and degradation in the gastrointestinal tract. Economically, the tested herbs in this trial are generally low in cost. More importantly, topical application of CDG effectively attenuated multiple dermatologic lesions associated with hand-foot skin reaction, acneiform eruption, and paronychia. Furthermore, since CDG is an instant herbal powder, it can be easily applied by all health care professionals. For these reasons, CDG may be considered for patients with targeted anticancer therapy-related dermatologic toxicities. However, the exact mechanisms underlying CDG's effect on reducing dermatologic toxicities are largely unknown. Based on TCM theory, CDG formula works on the principles of clearing heat and draining dampness, cooling, activating blood, and resolving stasis. Taken together, the above herbs could produce a beneficial effect on patients who experience dermatologic toxicities.
Some potential limitations in the current study should be noted. First, an important limitation is the short period of intervention. The relatively lower effect of CDG on acneiform eruption and paronychia may be correlated with shorter duration of intervention. A 10-day topical intervention may not be long enough for recovery of acneiform eruption and paronychia. Future studies should extend the treatment and follow-up duration. Second, our trial did not observe the preventive effect of CDG. As for the high prevalence of targeted anticancer therapy associated skin lesions (60-80\%), CDG may provide a useful role in prevention of skin toxicities, thereby increasing patient compliance to targeted anticancer therapies.

\section{Conclusions}

Our study suggests that topical application of CDG can effectively attenuate dermatologic toxicities associated with targeted anticancer therapy. The treatment effect of CDG was more pronounced in hand-foot skin reaction. A welldesigned RCT with large sample sizes is required to confirm the preventive effect of CDG in targeted anticancer therapy associated skin lesions. 

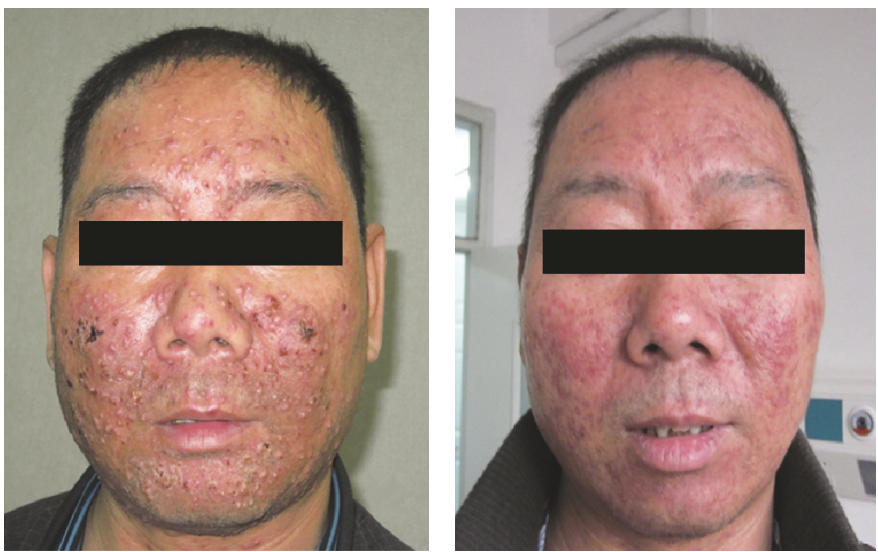

(a)
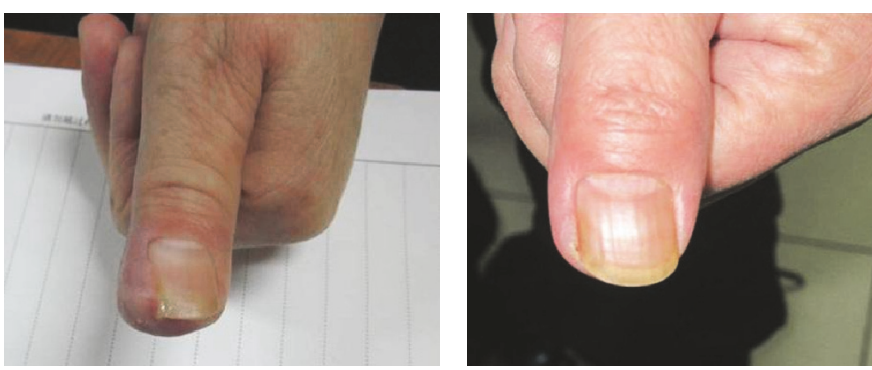

(b)
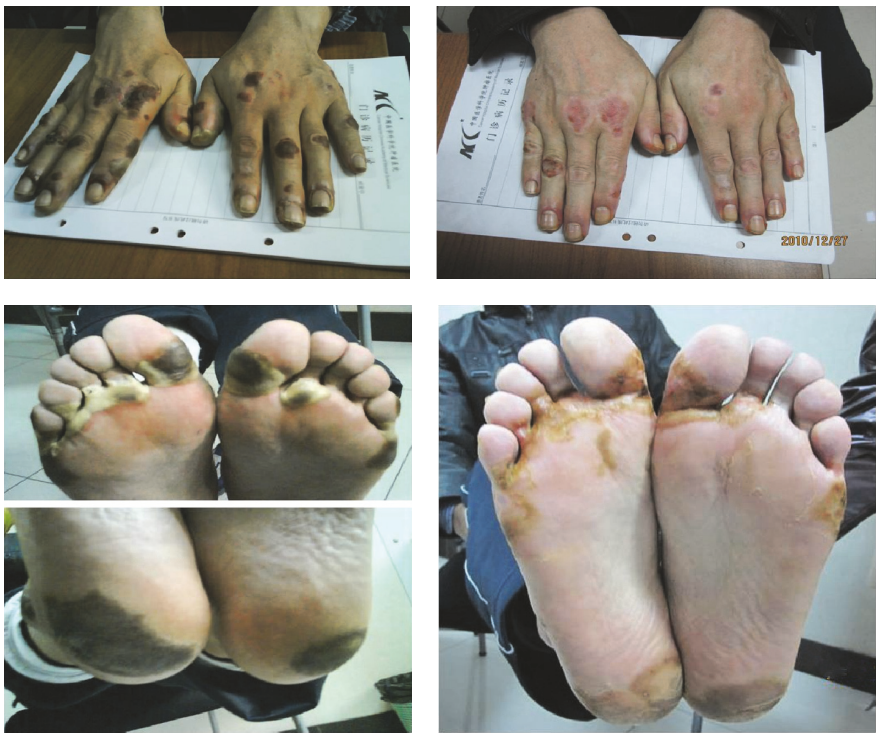

(c)

FIGURE 2: Three representative patients before and after treatment. (a) Acneiform eruption associated with Erlotinib in a patient with lung cancer. Before treatment (left), the face displayed swelling, itching, pain, purulent heads, and hemorrhage (Grade III). After 10 days of topical washes with CDG (right), the rash nearly healed. (b) Paronychia associated with Erlotinib in a patient with lung cancer. Before treatment (left), the nail exhibited pain, redness, and swelling (Grade III). After 10 days of topical washes with CDG (right), the tissues surrounding the nail recovered. (c) Hand-foot skin reaction associated with Sorafenib in a patient with hepatocellular carcinoma. Before treatment (left), skin areas on both hand and foot displayed redness, swelling, foaming, bleeding blister, and pain (Grade III). After 10 days of topical washes with CDG (right), the lesions were nearly healed. 
TABLE 3: Comparison of clinical effective rate between treatment groups.

\begin{tabular}{|c|c|c|c|c|}
\hline Group & Total effective rate & Treatment failure rate & Chi-square & $P$ value \\
\hline Compound Danxiong Granules $(n=67)$ & $52(77.61 \%)$ & $15(22.39 \%)$ & \multirow{2}{*}{23.55} & \multirow{2}{*}{$<0.0001$} \\
\hline Placebo $(n=33)$ & $9(27.27 \%)$ & $24(72.73 \%)$ & & \\
\hline
\end{tabular}

TABLE 4: Comparison of clinical effective rate on different types of skin lesions.

\begin{tabular}{|c|c|c|c|}
\hline Group & Total effective rate & Treatment failure rate & $P$ value \\
\hline Hand-foot skin reaction & & & $<0.0001$ \\
\hline Compound Danxiong Granules $(n=22)$ & $21(95.45 \%)$ & $1(4.55 \%)$ & \\
\hline Placebo $(n=11)$ & $3(27.27 \%)$ & $8(72.73 \%)$ & \\
\hline Acneiform eruption & & & 0.039 \\
\hline Compound Danxiong Granules $(n=26)$ & $18(69.23 \%)$ & $8(30.77 \%)$ & \\
\hline Placebo $(n=13)$ & $4(30.78 \%)$ & $9(69.23 \%)$ & \\
\hline Paronychia & & & 0.042 \\
\hline Compound Danxiong Granules $(n=19)$ & $13(68.42 \%)$ & $6(31.58 \%)$ & \\
\hline Placebo $(n=9)$ & $2(22.22 \%)$ & $7(77.78 \%)$ & \\
\hline
\end{tabular}

\section{Conflicts of Interest}

The authors declare that they have no conflicts of interest.

\section{References}

[1] H. Yasui and K. Imai, "Novel molecular-targeted therapeutics for the treatment of cancer," Anti-Cancer Agents in Medicinal Chemistry, vol. 8, no. 5, pp. 470-480, 2008.

[2] G. K. Dy and A. A. Adjei, "Understanding, recognizing, and managing toxicities of targeted anticancer therapies," A Cancer Journal for Clinicians, vol. 63, no. 4, pp. 249-279, 2013.

[3] J. Ulrich, J. T. Hartmann, W. Dörr, and S. Ugurel, "Skin toxicity of anti-cancer therapy," JDDG - Journal of the German Society of Dermatology, vol. 6, no. 11, pp. 959-977, 2008.

[4] C. Robert, V. Sibaud, C. Mateus, and B. S. Cherpelis, "Advances in the management of cutaneous toxicities of targeted therapies," Seminars in Oncology, vol. 39, no. 2, pp. 227-240, 2012.

[5] A. C. Rosen, E. C. Case, S. W. Dusza et al., "Impact of dermatologic adverse events on quality of life in 283 cancer patients: A questionnaire study in a dermatology referral clinic," American Journal of Clinical Dermatology, vol. 14, no. 4, pp. 327333, 2013.

[6] J. H. Borovicka, C. Calahan, M. Gandhi et al., "Economic burden of dermatologic adverse events induced by molecularly targeted cancer agents," Archives of Dermatology, vol. 147, no. 12, pp. 1403-1409, 2011.

[7] T. J. Lynch Jr., E. S. Kim, B. Eaby, J. Garey, D. P. West, and M. E. Lacouture, "Epidermal growth factor receptor inhibitorassociated cutaneous toxicities: An evolving paradigm in clinical management," Oncologist, vol. 12, no. 5, pp. 610-621, 2007.

[8] R. Gutzmer, A. Wollenberg, S. Ugurel, B. Homey, A. Ganser, and A. Kapp, "Cutaneous side effects of new antitumor drugs: clinical features and management," Deutsches Arzteblatt International, vol. 109, no. 8, pp. 133-140, 2012.

[9] L. Peuvrel and B. Dréno, "Dermatological toxicity associated with targeted therapies in cancer: optimal management," American Journal of Clinical Dermatology, vol. 15, no. 5, pp. 425-444, 2014.
[10] J. Koo and S. Arain, "Traditional chinese medicine for the treatment of dermatologic disorders," Archives of Dermatology, vol. 134, no. 11, pp. 1388-1393, 1998.

[11] J. Koo and R. Desai, "Traditional Chinese medicine in dermatology," Dermatologic Therapy, vol. 16, no. 2, pp. 98-105, 2003.

[12] L. Ye, Y. Jia, K. Ji et al., "Traditional Chinese medicine in the prevention and treatment of cancer and cancer metastasis (Review)," Oncology Letters, vol. 10, no. 3, pp. 1240-1250, 2015.

[13] J. Nie, C. Zhao, L. I. Deng et al., "Efficacy of traditional Chinese medicine in treating cancer," Biomedical Reports, vol. 4, pp. 314, 2016.

[14] M. Y. Hu and P. F. Chen, "Research progress in therapy of EGFRI-associated rash by traditional chinese medicine," Journal of Jiangxi University of Traditional Chinese Medicine, vol. 28, pp. 122-124, 2016.

[15] "National Cancer Institute (NCI). Common Terminology Criteria for Adverse Events v3.0 (CTCAE)," http://ctep.cancer.gov/ protocolDevelopment/electronic_applications/docs/ctcaev3.pdf.

[16] H. T. Eng and A. Chan, "Evidence-based treatment options for the management of skin toxicities associated with epidermal growth factor receptor inhibitors," Annals of Pharmacotherapy, vol. 43, no. 10, pp. 1658-1666, 2009.

[17] H. J. Guo and Z. J. Wang, "Traditional Chinese medicine intervention on skin toxicity induced by epidermal growth factor receptor inhibitor," Journal of Traditional Chinese Medicine, vol. 36, pp. 570-573, 2014.

[18] Y. Zhang, LC. Deng, and WS. Shen, "Antipruritic prescription treatment for acneiform eruption caused by targeted anticancer agents," Zhejiang Journal of Integrated Traditional Chinese, vol. 19, pp. 371-372, 2009.

[19] H. Y. Wang, C. Zou, H. J. Cui, W. Wang, S. C. Jiao, and J. L. Li, "EGFRIs-related rash treated with external Chinese medicinal with actions of clearing heat and draining dampness in 120 cases," Journal of Beijing University of Traditional Chinese Medicine, vol. 20, pp. 14-17, 2013.

[20] Y. H. Zhang, Y. Shen, L. Long, J. Yang, and T. Sun, "linical observation of treating rash induced by lung cancer targeted drug with Yangfei Xiaozhen Formula," China Journal of Traditional Chinese Medicine and Pharmacy, vol. 31, pp. 100-103, 2016. 


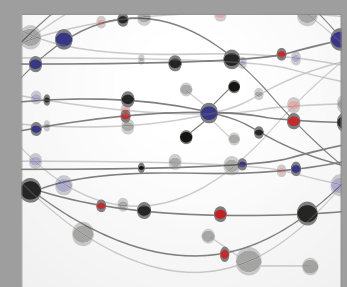

The Scientific World Journal
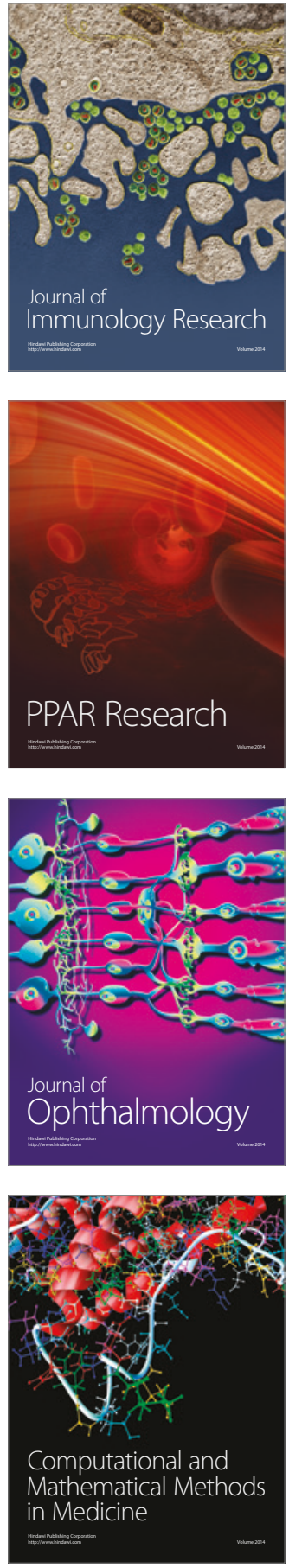

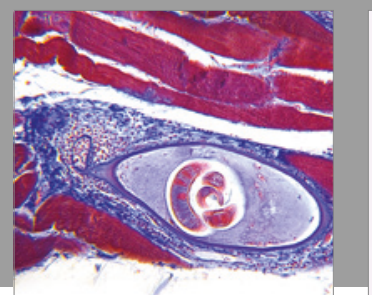

Gastroenterology Research and Practice
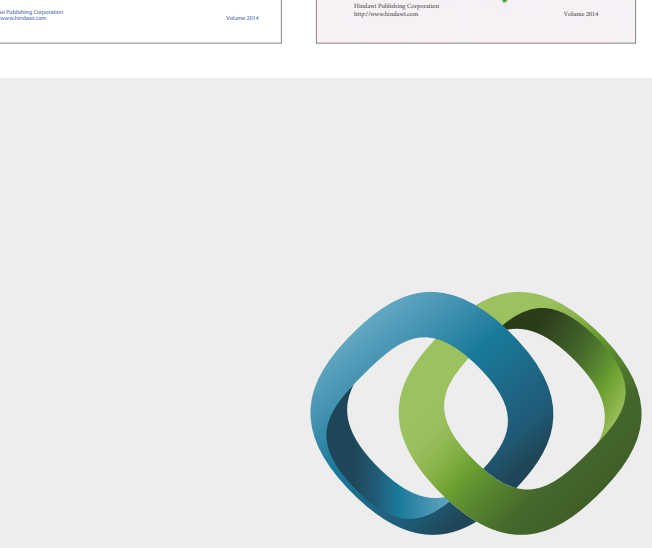

\section{Hindawi}

Submit your manuscripts at

https://www.hindawi.com
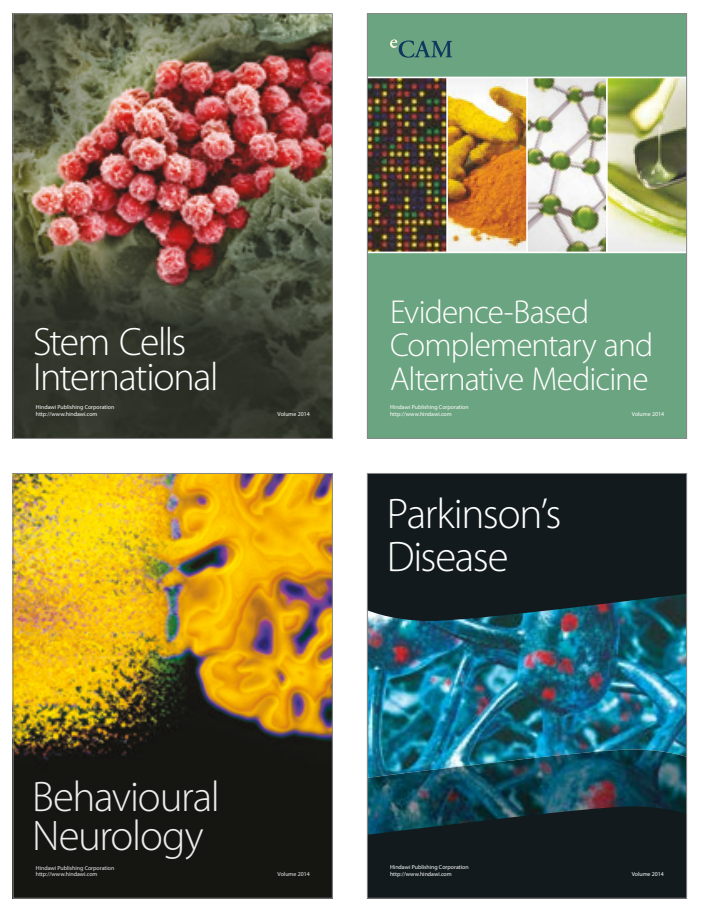
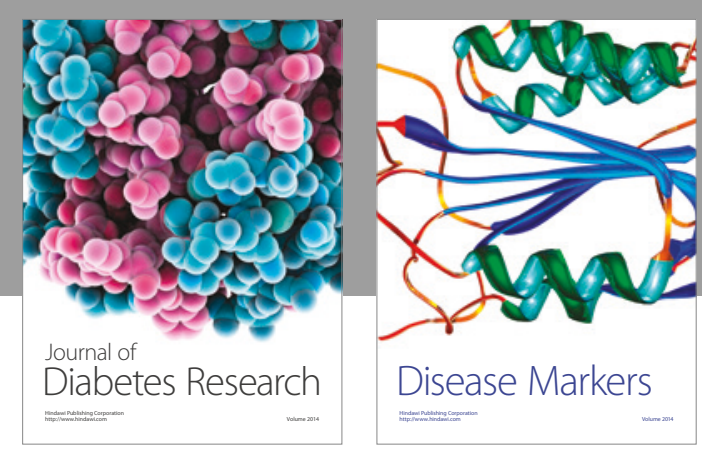

Disease Markers
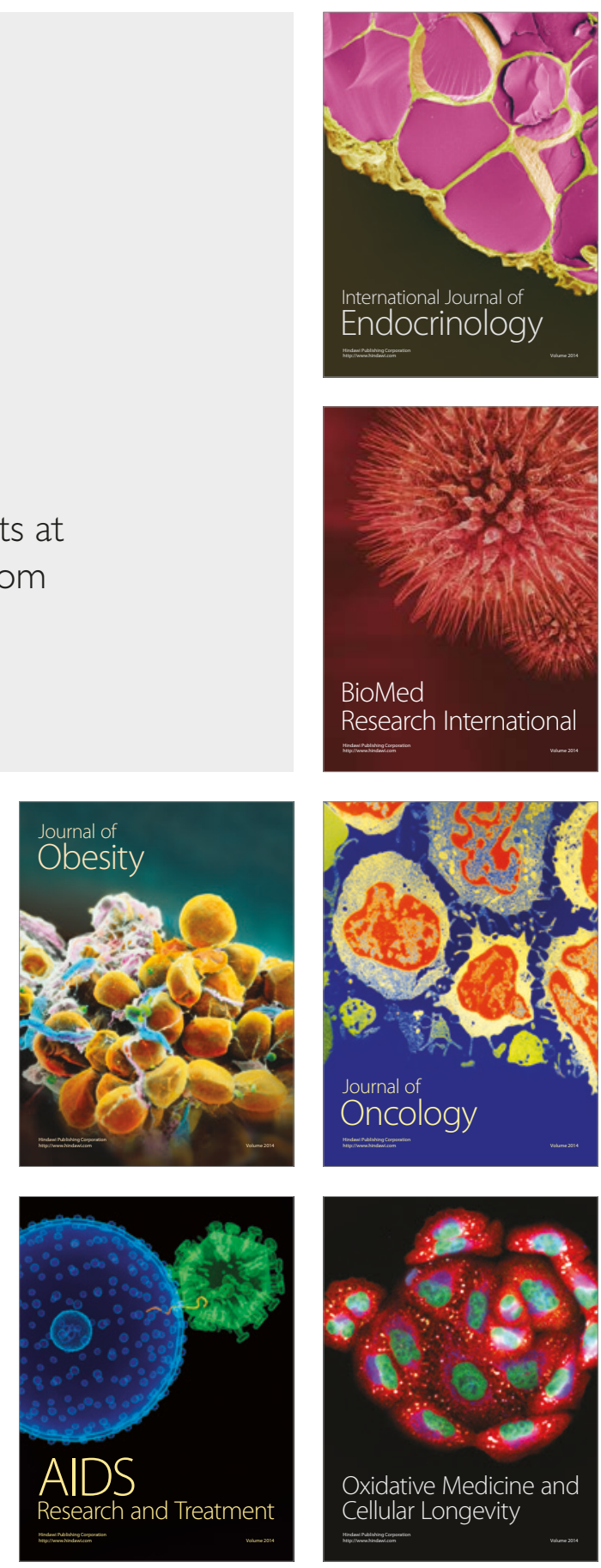